\title{
Dynamics of the Energy Relaxation and Decoherence of a Photon-Atom Bound State in an Anisotropic Photonic Crystal
}

\author{
Jing-Nuo Wu, ${ }^{1}$ Wen-Feng Hsieh, ${ }^{2}$ Hsin-Chien Huang, ${ }^{1}$ and Szu-Cheng Cheng ${ }^{1}$ \\ ${ }^{1}$ Department of Physics, Chinese Culture University, Taipei 11114, Taiwan \\ ${ }^{2}$ Department of Photonics and Institute of Electro-Optical Engineering, National Chiao Tung University, Hsinchu 71150, Taiwan
}

Correspondence should be addressed to Jing-Nuo Wu; jingnuowu@gmail.com and Szu-Cheng Cheng; sccheng@faculty.pccu.edu.tw

Received 21 May 2013; Accepted 16 September 2013

Academic Editor: Jörg Fink

Copyright (C) 2013 Jing-Nuo Wu et al. This is an open access article distributed under the Creative Commons Attribution License, which permits unrestricted use, distribution, and reproduction in any medium, provided the original work is properly cited.

An atom embedded inside photonic crystals can form a photon-atom bound state if the emission frequency of the excited atom is lying inside the photonic-band gap of photonic crystals. We studied the dynamics of the energy relaxation and decoherence of a QPAB, qubit made by a photon-atom bound state in photonic crystals. Dynamics of these measurements are solved analytically through the fractional calculus which has been shown to be appropriate mathematical method for the optical systems with nonMarkovian dynamics. From these dynamics, we find that the losses of energy, coherence, and information of a QPAB are inhibited. As compared with those qubits without forming photon-atom bound states, the energy relaxation and decoherence rates of these QPABs are strongly suppressed. Other systems suitable for realizing these properties are discussed.

\section{Introduction}

A central tenet in quantum computing is to create and manipulate qubits in a controllable way. Many novel methods have been proposed to generate controllable qubit states by changing the surrounding environment of the qubits [13]. When a qubit made by a two-level atom is embedded inside a structured reservoir with the photon density of state (DOS) different from that of free space, the dynamics of the qubit states are affected by the environment and exhibit non-Markovian behavior [4-6]. When strong coupling between the qubit and the structured reservoir is concerned, these non-Markovian dynamics are characterized by the memory effect that the information of the system is preserved for a long-time period. This characterization has recently constructed a number of experimental measures for exploring non-Markovianity in a variety of different systemenvironment models [7-11].

However, it is the interaction between a qubit and its surrounding environment that causes the energy relaxation and decoherence of the qubit. Since the quantum technologies rely on controlling quantum states with long-time coherence, it has become increasingly important to effectively suppress the energy relaxation and decoherence rates of quantum states. To achieve this goal, various schemes have been used to store quantum states with high fidelity through effectively controlling the energy relaxation and decoherence rates [1216]. Here we introduce a structured reservoir made by a photonic crystal $(\mathrm{PhC})$ whose photonic density of states (DOS) possesses a photonic-band gap (PBG). An excited atom embedded inside this reservoir is possible to form a photon-atom bound state [5] if the emitted frequency is lying inside the PBG. A qubit made by a photon-atom bound state in PhCs, which we abbreviate as a QPAB, exhibits some non-Markovian dynamics of the energy relaxation and decoherence of the QPAB. Systems with the forbidden bands in the structured reservoirs such as omnidirectional waveguides [17], tunable artificial crystals [18, 19], and frequency dispersive media [20] are also suitable for the realization of these non-Markovian dynamics.

In this paper, we shall show that the energy relaxation and decoherence rates of the QPAB are strongly suppressed and the long-time period of the coherence and information preservation of a qubit can be achieved via this structured reservoir. The steady states, shown by the polarization and von Neumann entropy of the QPAB, exhibit nonzero values 
for a very long-time period, indicating that the losses of energy and coherence of the QPAB are suppressed in this structured reservoir. The corresponding rates of energy relaxation and decoherence exhibit a fast decaying behavior that further manifests this suppressing phenomenon. This kind of structured reservoir provides qubits with a controllable environment that can preserve the qubit information for a long time.

The PhCs are ordered crystals with periodic variation of refractive index that show forbidden bands in their dispersion relation. Light-matter interactions in these periodical structures are drastically enhanced because they provide the photons with a reservoir with a threshold-like DOS near the forbidden band edges [21, 22]. As an excited atom is embedded (doped) in a $\mathrm{PhC}$, the emitted photon in this structured reservoir behaves very differently from that in free space. The appearance of photon-atom bound states [23-26], enhanced quantum interference [27], and non-Markovian effects [28-30] exist especially in this optical system. With the enhanced light-matter interactions, $\mathrm{PhCs}$ have been demonstrated theoretically and experimentally $[31,32]$ that they can be effectively used to inhibit spontaneous emission (SE) because of the existence of the photon-atom bound states.

In the studies on the photon-atom bound states in the doped PhCs, Singh controlled the SE cancellation of the PhCs doped with five-level nanoparticles by moving the resonance energies between the energy band and energy gap where bound states are formed [25]. Generalizing the properties of the PhCs to the dispersive media, Rupasov and Singh studied the quantum electrodynamics of a single two-level atom placed in a frequency dispersive medium (DM) and found a polariton-atom bound state with an eigenfrequency lying within the gap of the polariton spectrum [20,23]. This generalization arises the intriguing question about whether the quantum optical phenomena observed in PhCs can also be expected in dispersive media and vice versa. We discuss this question through comparing our findings with the results found in the dispersive media in Section 2.3.

When a practical three-dimensional (3D) $\mathrm{PhC}$ is considered, the dispersion relation near the forbidden band edge exhibits a directional-dependent property and thus has been expressed as a vector form by the effective-mass approximation [33]. This 3D material with the anisotropic band structure possesses a DOS different from the free-space one and proportional to $\sqrt{\omega-\omega_{c}} \Theta\left(\omega-\omega_{c}\right)$, where $\omega_{c}$ is the band-edge frequency and $\Theta\left(\omega-\omega_{c}\right)$ is the Heaviside step function. States with frequencies lying below the band edge $\omega_{c}$ are forbidden. In this forbidden region, the emission of a photon from a qubit, constructed by an excited twolevel atom, is inhibited, so a photon-atom bound state [5] is formed when the qubit interacts with its surrounding reservoir strongly.

This paper is organized as follows. In Section 2, the theoretical model for the system of a qubit in an anisotropic $\mathrm{PhC}$ is depicted through the Hamiltonian and the wave function. The kinetic equation of the qubit can be written as a fractional Langevin equation and solved by the fractional calculus, a mathematical method appropriate for

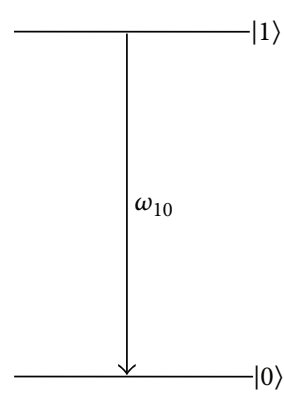

(a)

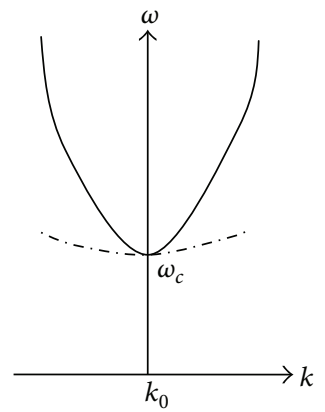

(b)

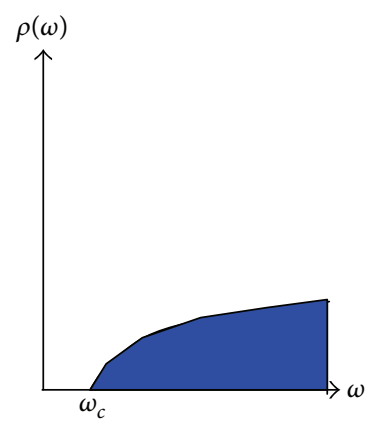

(c)

Figure 1: (a) A qubit with excited state $|1\rangle$ and ground state $|0\rangle$. The transition frequency $\omega_{10}$ is nearly resonant with the frequency range of the $\mathrm{PhC}$ reservoir. (b) Directional dependent dispersion relations near band edge expressed by the effective-mass approximation with the edge frequency $\omega_{c}$ as solid and dashed curves. (c) Photon DOS $\rho(\omega)$ of the anisotropic $\mathrm{PhC}$ reservoir exhibiting forbidden photon mode below the edge frequency $\omega_{c}$.

solving the system with non-Markovian dynamics $[6,28]$, to construct the reduced density matrix of the qubit. Dynamics of the energy relaxation rate, polarization, and von Neumann entropy are calculated and analyzed based on the elements of this density matrix. Systems of omnidirectional waveguides, tunable artificial crystals, and dispersive media suitable for realizing this property are briefly discussed at the end of this section. Finally, we summarize our results in Section 3.

\section{Dynamics of a Qubit in an Anisotropic PhC}

When we consider a system of a qubit formed by an excited atom embedded inside a structured reservoir of an anisotropic PhC shown in Figure 1, the Hamiltonian can be expressed as

$$
\begin{aligned}
H= & \hbar \omega_{10}|1\rangle\langle 1|+\sum_{\vec{k}} \hbar \omega_{\vec{k}} a_{\vec{k}}^{+} a_{\vec{k}} \\
& +i \hbar \sum_{\vec{k}} g_{\vec{k}}\left(|0\rangle\langle 1| \otimes a_{\vec{k}}^{+}+\text {h.c. }\right) .
\end{aligned}
$$

Here $\omega_{10}$ is the qubit transition frequency between the excited state $|1\rangle$ and ground state $|0\rangle$. The photon mode with frequency $\omega_{\vec{k}}$ is created and annihilated by the operators $a_{\vec{k}}^{+}$and $a_{\vec{k}}$. Coupling strength associated with the qubit and 
the photon mode with frequency $\omega_{\vec{k}}$ is specified by $g_{\vec{k}}=$ $\left(\omega_{10} d_{10} / \hbar\right)\left[\hbar / 2 \epsilon_{0} \omega_{\vec{k}} V\right]^{1 / 2} \widehat{e}_{\vec{k}} \cdot \widehat{u}_{d}$ with the fixed qubit dipole moment $\vec{d}_{10}=d_{10} \widehat{u}_{d}$, sample volume $V$, dielectric constant $\epsilon_{0}$, and polarization unit vector $\widehat{e}_{\vec{k}}$ of the photon mode with frequency $\omega_{\vec{k}}$. If we use the coordinate $(\theta, \phi)$ on the Bloch sphere to parameterize the state of the qubit with $(0, \phi)$ for the excited state and $(\pi, \phi)$ for the ground state, then the initial state of the total system can be written as $|\psi(0)\rangle=\left[e^{i \phi_{0}} \cos \left(\theta_{0} / 2\right)|1\rangle+\sin \left(\theta_{0} / 2\right)|0\rangle\right] \otimes\left|\mathbf{0}_{\vec{k}}\right\rangle$ with the initial coordinate $\left(\theta_{0}, \phi_{0}\right)$ on the Bloch sphere. Here the vacuum state and one-excitation state of the $\mathrm{PhC}$ reservoir are expressed as $\left|\mathbf{0}_{\vec{k}}\right\rangle=\left|0_{1}, 0_{2}, \ldots, 0_{\vec{k}}, 0_{\vec{k}+1}, \ldots\right\rangle$ and $\left|\mathbf{1}_{\vec{k}}\right\rangle=\left|0_{1}, 0_{2}, \ldots 0_{\vec{k}-1}, 1_{\vec{k}}, 0_{\vec{k}+1}, \ldots\right\rangle$, respectively. As the system evolves, the quantum state of the system in the single photon sector can be written as

$$
\begin{aligned}
|\psi(t)\rangle= & {\left[u_{p}(t) e^{-i \omega_{10} t} e^{i \phi_{0}} \cos \left(\frac{\theta_{0}}{2}\right)|1\rangle\right.} \\
& \left.+u_{d}(t) \sin \left(\frac{\theta_{0}}{2}\right)|0\rangle\right] \otimes\left|\mathbf{0}_{\vec{k}}\right\rangle \\
& +\sum_{\vec{k}} C_{\vec{k}}(t) e^{-i \omega_{\vec{k}} t}|0\rangle \otimes\left|\mathbf{1}_{\vec{k}}\right\rangle
\end{aligned}
$$

with initial condition $u_{p}(0)=1, u_{d}(0)=1$, and $C_{\vec{k}}(0)=0$. Here $u_{p}(t)$ and $u_{d}(t)$ stand for the excited-state and groundstate probability amplitudes of the qubit with the vacuum state of the $\mathrm{PhC}$ reservoir while $C_{\vec{k}}(t)$ is the probability amplitude of the photonic state at $\vec{k}$. This quantum state can also be expressed as $|\psi(t)\rangle=\left\{e^{i \phi(t)} \cos [\theta(t) / 2]|1\rangle+\sin [\theta(t) / 2]|0\rangle\right\} \otimes$ $\left|\mathbf{0}_{\vec{k}}\right\rangle+\sum_{\vec{k}} C_{\vec{k}}(t)|0\rangle \otimes\left|\mathbf{1}_{\vec{k}}\right\rangle$ with the time-dependent Bloch parameters $[\theta(t), \phi(t)]$ when we discuss the change of the qubit's state on the Bloch sphere.

By substituting the quantum state in (2) for the timedependent Schrödinger equation, we obtain the equations of motion for the amplitudes as

$$
\begin{gathered}
\frac{d}{d t} u_{p}(t)=-\frac{1}{e^{i \phi_{0}} \cos \left(\theta_{0} / 2\right)} \sum_{\vec{k}} g_{\vec{k}} C_{\vec{k}}(t) e^{-i \Omega_{\vec{k}} t}, \\
\frac{d}{d t} C_{\vec{k}}(t)=g_{\vec{k}} u_{p}(t) e^{i \phi_{0}} \cos \left(\frac{\theta_{0}}{2}\right) e^{i \Omega_{\vec{k}} t}, \\
\frac{d}{d t} u_{d}(t)=0
\end{gathered}
$$

with detuning frequency $\Omega_{\vec{k}}=\omega_{\vec{k}}-\omega_{10}$. The last equation of motion yields $u_{d}(t)=u_{d}(0)=1$ meaning that the groundstate probability of a qubit will not evolve with time, that is, a constant of time. The other two equations can be combined as $(d / d t) u_{p}(t)=-\int_{0}^{t} G(t-\tau) u_{p}(\tau) d \tau$ with the memory kernel $G(t-\tau)=\sum_{\vec{k}} g_{\vec{k}}^{2} e^{-i \Omega_{\vec{k}}(t-\tau)}$. This equation of motion reveals that the time dependence of the qubit state is determined by all previous states, called the long-time memory effect or non-Markovian behavior, through the memory kernel. As the qubit is put in the free space, the memory kernel becomes a Dirac delta function $G(t-\tau) \propto \delta_{d}(t-\tau)$ corresponding to continuous photon DOS. In this case, the reservoir manifests its memory effect only at an instant time $\tau=t$ which leads to the excited amplitude of the qubit decaying exponentially with time. This Markovian result in free space manifests that the qubit loses all memory of its past and decays quickly to its ground state.

For the anisotropic PhC reservoir discussed here, the memory kernel manifests its memory effect within the entire time interval $(0, t)$ through $G(t-\tau)=\left(\left(\beta^{1 / 2} / f^{3 / 2}\right) /(\sqrt{\pi}(t-\right.$ $\left.\left.\tau)^{3 / 2}\right)\right) e^{-i[3 \pi / 4-\delta(t-\tau)]}$ with the coupling constant $\beta^{1 / 2}=$ $\left(\omega_{10}^{2} d_{10}^{2} \sqrt{\omega_{c}}\right) /\left(16 \pi \epsilon_{0} \hbar c^{3}\right)$ and the detuning frequency $\delta=$ $\omega_{10}-\omega_{c}$ of the qubit transition frequency $\omega_{10}$ from the band edge frequency $\omega_{c}$ [6]. This memory kernel is derived from the anisotropic dispersion relation of a practical threedimensional PhC. Near the band edge frequency $\omega_{c}$, the dispersion relation has a vector form and could be expressed by the effective-mass approximation as [34] $\omega_{\vec{k}} \approx \omega_{c}+A\left(\vec{k}-\vec{k}_{c}\right)^{2}$, where the curvature $A \cong f \omega_{c} / k_{c}^{2}=f c^{2} / \omega_{c}$ signifies its different values in different directions through the scaling factor $f$. This anisotropic dispersion relation leads to the memory kernel expressed by the cut-off photon DOS $\rho(\omega)$ through $G(t-\tau)=\left(\omega_{10}^{2} d_{10}^{2} / 4 \epsilon_{0} \hbar\right) \int_{0}^{\infty} d \omega(\rho(\omega) / \omega) e^{-i\left(\omega-\omega_{10}\right)(t-\tau)}$ with $\rho(\omega)=\left(1 / 4 \pi^{2}\right) \sqrt{\left(\left(\omega-\omega_{c}\right) / A^{3}\right)} \Theta\left(\omega-\omega_{c}\right)$ and the Heaviside step function $\Theta\left(\omega-\omega_{c}\right)$ charactering the cut-off behavior. With this memory kernel for the anisotropic $\mathrm{PhC}$ reservoir and making the transformation $u_{p}(t)=e^{i \delta t} U_{p}(t)$, the equation of motion becomes

$$
\frac{d}{d t} U_{p}(t)+i \delta U_{p}(t)=\frac{\beta^{1 / 2} e^{i \pi / 4}}{\sqrt{\pi} f^{3 / 2}} \int_{0}^{t} \frac{U_{p}(\tau)}{(t-\tau)^{3 / 2}} d \tau .
$$

Conventionally, this integrodifferential equation is solved through Laplace transform which leads to the fractal phenomenon of the system from this memory kernel in Laplace image [35]. This fractal phenomenon would result in the stochastic nature of the dynamical behavior of the system which appears as the non-Markovian dynamics. These nonMarkovian dynamics can be solved accurately through fractional calculus which we have shown its appropriateness by comparing the obtained results with the experimental ones and finding their consistence $[6,28]$.

In the following, we shall use fractional calculus to solve the non-Markovian dynamics of the system analytically. In particular, the fractional time derivative, one of the operators of fractional calculus, is used to express the integral term of the kinetic equation (4). In the well-known RiemannLiouville definition, the fractional time derivative operator $d^{v} / d t^{v}$ is expressed as [36]

$$
\frac{d^{\nu}}{d t^{\nu}} f(t)=\frac{1}{\Gamma(n-\nu)} \frac{d^{n}}{d t^{n}} \int_{a}^{t} \frac{f(\tau)}{(t-\tau)^{\nu-n+1}} d \tau
$$

for $n-1 \leq v<n$ and $\Gamma(x)$ being the gamma function. Through expressing the right-hand-side term of (4) as the fractional time derivative operator with order $n=1 / 2$ and applying the appropriate fractional operations to this kinetic equation, we 
arrive at the fractional kinetic equation of this qubit-reservoir interacting system as

$$
\frac{d^{1 / 2}}{d t^{1 / 2}} U_{p}(t)+i \delta \frac{d^{-1 / 2}}{d t^{-1 / 2}} U_{p}(t)+\frac{2 \beta^{1 / 2} e^{i \pi / 4}}{f^{3 / 2}} U_{p}(t)=\frac{t^{-1 / 2}}{\sqrt{\pi}} .
$$

The fractional time derivative in this fractional differential equation indicates a subordinated stochastic process directing to a stable probability distribution [37]. The evolution equation governing the future of the qubit is expressed as the form of a fractional Langevin equation because of its interaction with the $\mathrm{PhC}$ reservoir. Our aim of studying the quantum dynamics of relaxation, decoherence, and entropy for the qubit system can be achieved by solving this equation through the Laplace transform for the fractional operators. The basic formula used here is

$$
\begin{aligned}
L\left\{\frac{d^{\nu}}{d t^{\nu}} f(t)\right\} & \equiv \int_{0}^{\infty} e^{-s t} \frac{d^{\nu}}{d t^{\nu}} f(t) d t \\
& =s^{\nu} L\{f(t)\}-\sum_{m=0}^{n-1} s^{m}\left[\frac{d^{\nu-m-1}}{d t^{\nu-m-1}} f(t)\right]_{t=0}
\end{aligned}
$$

with $s$ denoting the Laplace variable. The procedure of performing the Laplace transform on the fractional Langevin equation leads to $\widetilde{U}_{p}(s)=1 /\left(s+i \delta+2 \beta^{1 / 2} e^{i \pi / 4} s^{1 / 2} / f^{3 / 2}\right)$, where $\widetilde{U}_{p}(s)$ is the Laplace transform of $U_{p}(t)$. This equation can be further expressed as a sum of partial fractions as the roots of the indicial equation $Y^{2}+2 \beta^{1 / 2} e^{i \pi / 4} Y / f^{3 / 2}+i \delta=$ 0 are found with the variable $s^{1 / 2}$ having been converted into $Y$. As $\beta / f^{3} \neq \delta$, we have the different roots of the indicial equation which leads to the partial-fractional form of $\widetilde{U}_{p}(s)=\left[\left(1 /\left(\sqrt{s}-Y_{1}\right)\right)-\left(1 /\left(\sqrt{s}-Y_{2}\right)\right)\right]\left(1 /\left(Y_{1}-Y_{2}\right)\right)$ with $Y_{1}=e^{i \pi / 4}\left(-\left(\beta^{1 / 2} / f^{3 / 2}\right)+\sqrt{\left(\beta / f^{3}\right)-\delta}\right)$ and $Y_{2}=$ $e^{i \pi / 4}\left(-\left(\beta^{1 / 2} / f^{3 / 2}\right)-\sqrt{\left(\beta / f^{3}\right)-\delta}\right)$. For $\beta / f^{3}=\delta$, the indicial equation has degenerate root leading to $\widetilde{U}_{p}(s)=1 /(\sqrt{s}+$ $\left.\left(\beta^{1 / 2} e^{i \pi / 4} / f^{3 / 2}\right)\right)^{2}$.

The inverse Laplace transform of these partial-fractional forms cannot be found in the conventional mathematical table because the power of the variable $s$ is not an integer. For the fractional power of $s$, we can find its inverse Laplace transform in the book of fractional calculus [38] as $L^{-1}\left\{1 /\left(s^{\nu}-\right.\right.$ $a)\}=\sum_{j=1}^{q} a^{j-1} E_{t}\left(j v-1, a^{q}\right)$ and $L^{-1}\left\{1 /\left(s^{\nu}-a\right)^{2}\right\}=$ $\sum_{j=1}^{q} \sum_{m=1}^{q} a^{j+m-2}\left\{t E_{t}\left((j+m) v-2, a^{q}\right)-[(j+m) v-2] E_{t}((j+\right.$ m) $\left.\left.v-1, a^{q}\right)\right\}$ with $v=1,1 / 2,1 / 3, \ldots$ and $q=1,2, \ldots, 1 / \nu$. Here we name the two-parameter function $E_{t}(\alpha, a)$ as the fractional exponential function with variable $t$, order $\alpha$, and constant $a$. It is defined as the fractional derivative of an ordinary exponential function $E_{t}(\alpha, a) \equiv\left(d^{-\alpha} / d t^{-\alpha}\right) e^{a t}=$ $t^{\alpha} \sum_{n=0}^{\infty}\left((a t)^{n} / \Gamma(\alpha+n+1)\right)$ with the derivative formula $\left(d^{\mu} / d t^{\mu}\right) E_{t}(\alpha, a)=E_{t}(\alpha-\mu, a)$. The functional form of this fractional exponential function $E_{t}(\alpha, a)$ will not be changed after being performed on derivative operator with fractional or integral order. A linear combination of the fractional exponential functions is a potential solution of the fractional differential equation. By applying these inverse Laplace transforms to the partial-fractional forms of $\widetilde{U}_{p}(s)$, we obtain the analytical solution of the fractional differential equation as

$$
\begin{aligned}
U_{p}(t)= & \frac{1}{2 e^{i \pi / 4} \sqrt{\beta / f^{3}-\delta}} \\
& \times\left[Y_{1}^{2} E_{t}\left(1 / 2, Y_{1}^{2}\right)-Y_{2}^{2} E_{t}\left(1 / 2, Y_{2}^{2}\right)\right. \\
& \left.+Y_{1} e^{Y_{1}^{2} t}-Y_{2} e^{Y_{2}^{2} t}\right], \\
U_{p}(t)= & -2 \frac{\beta^{3 / 2} e^{i 3 \pi / 4}}{f^{9 / 2}} t E_{t}\left(\frac{1}{2}, \frac{i \beta}{f^{3}}\right) \\
& -\frac{\beta^{1 / 2} e^{i \pi / 4}}{f^{3 / 2}} E_{t}\left(\frac{1}{2}, \frac{i \beta}{f^{3}}\right)+\left(1+\frac{2 i t \beta}{f^{3}}\right) e^{i \beta t / f^{3}} \\
& -2 \frac{\beta^{1 / 2} e^{i \pi / 4}}{f^{3 / 2} \sqrt{\pi}} t^{1 / 2}
\end{aligned}
$$

for $\beta / f^{3} \neq \delta$ and $\beta / f^{3}=\delta$, respectively. Here we have applied the recursion relation of the fractional exponential function $E_{t}(\nu, a)=a E_{t}(\nu+1, a)+t^{\nu} / \Gamma(\nu+1)$ to these analytical expressions. The time evolution of the wave function is thus obtained as

$$
\begin{aligned}
& |\psi(t)\rangle \\
& =\left[u_{p}(t) e^{i \phi_{0}} \cos \left(\frac{\theta_{0}}{2}\right)|1\rangle+u_{d}(t) \sin \left(\frac{\theta_{0}}{2}\right)|0\rangle\right] \otimes\left|\mathbf{0}_{\vec{k}}\right\rangle \\
& \quad+\sum_{\vec{k}} C_{\vec{k}}(t)|0\rangle \otimes\left|\mathbf{1}_{\vec{k}}\right\rangle
\end{aligned}
$$

with $u_{p}(t)=e^{i \delta t} U_{p}(t)$ expressed through (8a) and (8b), $u_{d}(t)=u_{d}(0)=1$, and $\sum_{\vec{k}}\left|C_{\vec{k}}(t)\right|^{2}=1-\left[u_{p}(t) \cos \left(\theta_{0} / 2\right)\right]^{2}-$ $\left[\sin \left(\theta_{0} / 2\right)\right]^{2}$.

By definition, the reduced density matrix of the qubit can be directly obtained from this wave function through tracing over the reservoir degrees of freedom. It gives

$$
\begin{aligned}
\hat{\rho}(t) & =\left(\begin{array}{cc}
\left|u_{p}(t)\right|^{2} \cos ^{2}\left(\frac{\theta_{0}}{2}\right) & \frac{1}{2} u_{p}^{*}(t) e^{-i \phi_{0}} \sin \left(\theta_{0}\right) \\
\frac{1}{2} u_{p}(t) e^{i \phi_{0}} \sin \left(\theta_{0}\right) & 1-\left|u_{p}(t)\right|^{2} \cos ^{2}\left(\frac{\theta_{0}}{2}\right)
\end{array}\right) \\
& \equiv\left(\begin{array}{ll}
\rho_{11}(t) & \rho_{10}(t) \\
\rho_{01}(t) & \rho_{00}(t)
\end{array}\right)
\end{aligned}
$$

with the initial one

$$
\hat{\rho}(0)=\left(\begin{array}{cc}
\cos ^{2}\left(\theta_{0} / 2\right) & (1 / 2) e^{-i \phi_{0}} \sin \left(\theta_{0}\right) \\
(1 / 2) e^{i \phi_{0}} \sin \left(\theta_{0}\right) & \sin ^{2}\left(\theta_{0} / 2\right)
\end{array}\right) .
$$

The elements in this matrix are associated with the polarization and probabilities of the qubit. In the following, we will 
study the quantum measurements of the energy relaxation, decoherence, and von Neumann entropy based on these elements.

2.1. Energy-Relaxation Rate. As the qubit spontaneously emits a photon, the probability amplitude of the qubit undergoes a quantum damping and becomes small. This amplitude damping associated with the energy dissipation of the qubit leads to the flow of energy from the qubit into the environment through the emitted photon. How the energy flows from the initially prepared qubit can be observed from the excited-state probability of the qubit $P(t)$ through $P(t)=$ $\left|u_{p}(t)\right|^{2} \cos ^{2}\left(\theta_{0} / 2\right)=\left|U_{p}(t)\right|^{2}\left(\theta_{0}=0\right)$ shown in Figure $2(\mathrm{a})$. The probability amplitude of a usual qubit $\left[\delta / \beta=\left(\omega_{10}{ }^{-}\right.\right.$ $\left.\omega_{c}\right) / \beta>0$ ] decays to zero quickly and the qubit has a shortlife time. For the QPAB $[\delta / \beta<0]$, the probability dynamics exhibit decay and oscillatory behavior before reaching a steady nonzero value, indicating that the energy flow from the QPAB to the PhC reservoir is inhibited and a photonatom bound state is formed. The rate of this energy flow can be measured through the energy-relaxation rate $\Gamma_{\text {relax. }}(t)=$ $-\dot{\rho}_{11}(t) / \rho_{11}(t)=-\left[\left(\dot{U}_{p}(t) / U_{p}(t)\right)+\left(\dot{U}_{p}^{*}(t) / U_{p}^{*}(t)\right)\right]$ with $U_{p}^{*}(t)$ being the complex conjugate of $U_{p}(t)$ in $(8 \mathrm{a})$ and $(8 \mathrm{~b})$. In Figure 2(b), we find that the energy-relaxation rates of QPABs and usual qubits decay to zero very quickly and slowly, respectively.

Combining the dynamical behavior of the probability amplitude and energy-relaxation rate of a qubit, we find that the QPAB releases and preserves some of its energy at the very beginning and at the end, respectively. The quick stop of releasing energy results from the formation of the bound state between the qubit and the localized photon. The remaining energy continuously excites the atom leading to the nondecaying dynamics of the probability amplitude in Figure 2(a) and quick decaying of the energy-relaxation rate in Figure 2(b). Note that, as the emitted frequency of the QPAB is detuned deep inside the forbidden band region $(\delta / \beta=-5$ and -10$)$, the energy-relaxation rates exhibit negative values in the initial period of relaxation. This negative energy-relaxation rate reveals that the qubit can regain its energy in the $\mathrm{PhC}$ reservoir. That is, we observe the released energy from the QPAB flowing back to the QPAB during a certain time interval. This character of the long-time memory effect also manifests the controllable relaxation of a qubit inside the structured reservoir. On the other hand, for a usual qubit, the energy-relaxation rate decays slowly and remains a nonzero value for a long time in Figure 2(b). A usual qubit releases its energy continuously and the emitted photon from the qubit carries the energy and information away.

When we use a Bloch vector in the Bloch sphere to express the qubit state, the amplitude damping will perform a transformation on the components of the Bloch vector with the values related to the probability of losing the emitted photon [39]. For the Markovian system of an usual qubit, the large probability of losing the emitted photon will convert the Bloch vector toward the ground-state point on the Bloch sphere. The small probability of losing the emitted photon

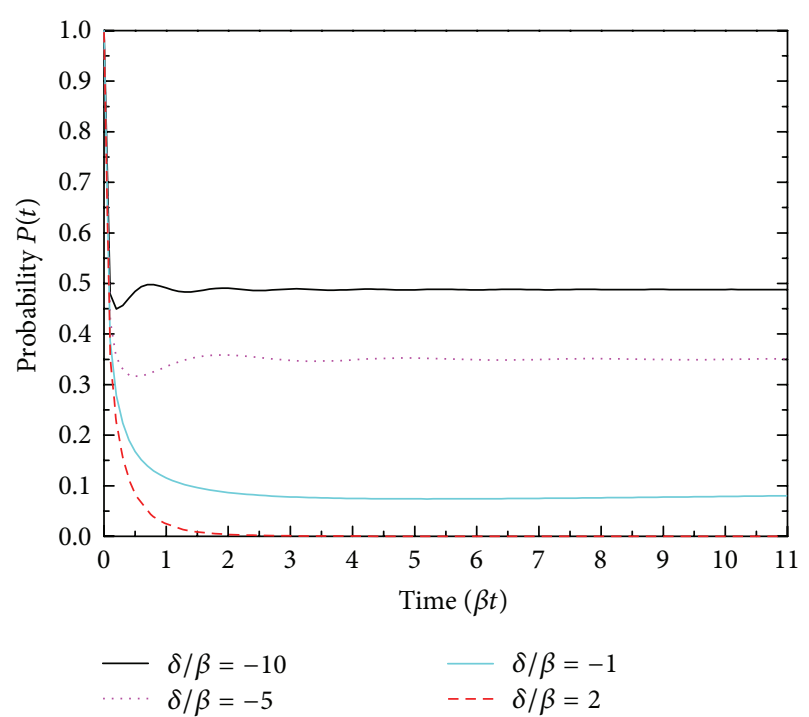

(a)

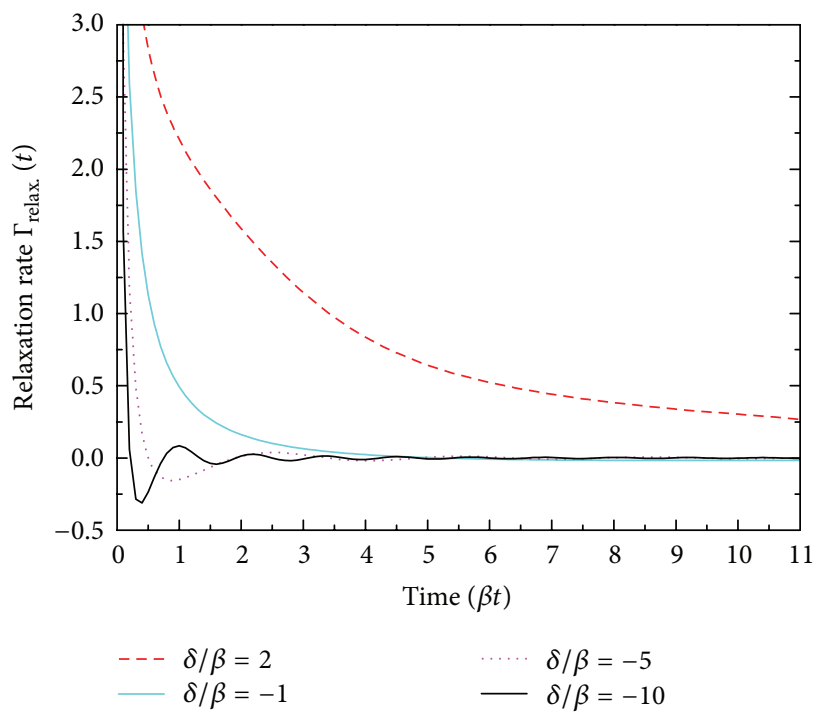

(b)

Figure 2: Dynamics of (a) the qubit's excited-state probability $P(t)$ and (b) relaxation rate $\Gamma_{\text {relax. }}(t)$ of the qubit with different detuning frequencies $\delta / \beta=\left(\omega_{10}-\omega_{c}\right) / \beta$ from the band edge frequency $\omega_{c}$ of the $\mathrm{PhC}$ reservoir.

in the non-Markovian system of a QPAB will convert the Bloch vector pointing towards the nonzero surface area near the ground-state point on the Bloch sphere. The contracting effect of the amplitude damping on the Bloch vector is strongly suppressed in the non-Markovian system.

2.2. Decoherence and von Neumann Entropy. As a qubit couples with the reservoir, the polarization of the qubit is randomized due to the reservoir. The coherence phase of a qubit determined by the qubit polarization is also randomized and leads to the quantum decoherence. As the qubit undergoes the polarization randomization through this quantum 

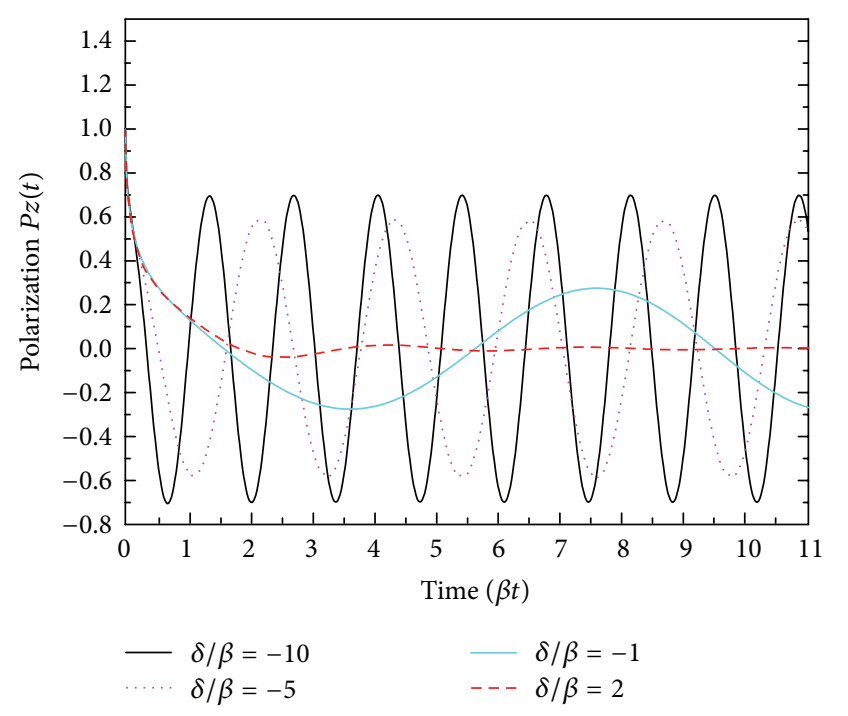

(a)

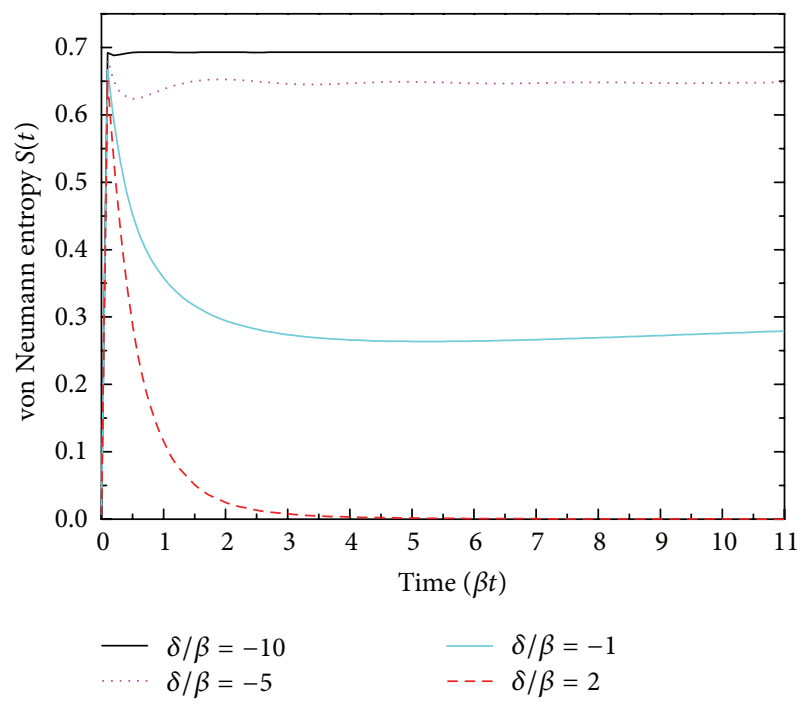

(b)

FIgURe 3: (a) Polarization $P z(t)=\rho_{10}(t)+\rho_{01}(t)$ and (b) von Neumann entropy $S(t)=-\operatorname{Tr}[\hat{\rho}(t) \log \hat{\rho}(t)]=-\lambda_{+} \log \lambda_{+}-$ $\lambda_{-} \log \lambda_{-}$of the non-Markovian $(\delta / \beta<0)$ and Markovian $(\delta / \beta=2)$ systems.

decoherence, the off-diagonal elements of the reduced density matrix will decay with time. The completely loss of the quantum coherence of one qubit will lead to the occurrence of entanglement sudden death (ESD) in the multiple-qubit system. That is, the time period in which the entanglement could be usefully exploited in the multiqubit system is limited. This limitation is related to the dynamics of the qubit polarization shown in Figure 3(a) by defining the polarization of a qubit as $P z(t)=\rho_{10}(t)+\rho_{01}(t)=\operatorname{Re}\left[e^{i \delta t} U_{p}(t)\right]$ (if $\phi_{0}=0$, $\left.\theta_{0}=\pi / 2\right)$.

For a usual qubit with $\delta / \beta=2$, the polarization of the qubit exhibits a fast damping behavior implying that the qubit loses all its polarization in the initial period of time with $\beta t<4$. This polarization damping results from the qubit scattered inelastically by incoherent background fields from the reservoir. The inelastic scattering randomizes the orientation of the qubit polarization and leads to the decay of the off-diagonal elements of the density matrix. The fast damping of the qubit polarization reveals that the probability of the qubit being scattered by incoherent photons of the reservoir is large in the Markovian system. The photons from the allowed band with a continuous DOS (see Figure 1(c)) provide the free-space-like environment to destroy the qubit.

On the other hand, the polarization of a QPAB exhibits a nondecaying oscillation. The QPAB loses some of its polarization at the very beginning and then preserves the remaining polarization shown by the steady oscillation of its polarization. This steady polarization ensures that the probability of the QPAB scattered by incoherent photons of the reservoir is greatly lowered in the non-Markovian system. The PBG shown in the photon DOS of the PhC reservoir (see Figure $1(\mathrm{c})$ ) is expected to lower this scattering probability and to lead to the preservation of phase information of the qubit through the steady polarization. The QPAB with a larger amplitude of polarization has less loss of coherence information to the reservoir.

Entropy, a key concept of quantum information theory, measures how much information exists in a state of a physical system. The amount of information is changed with respect to the correlation of a state and the reservoir. The correlation between the environment and a state will transform the initially purified state into a finally mixed state where the state and its environment are entangled. We can thus estimate the correlation between the qubit and reservoir through the entropy of the quantum system, that is, von Neumann entropy. The von Neumann entropy for a quantum state with $\hat{\rho}(t)$ is defined as $S(t)=-\operatorname{Tr}[\hat{\rho}(t) \log \hat{\rho}(t)]=-\sum_{i} \lambda_{i} \log \lambda_{i}$ with $\lambda_{i}$ being the eigenvalues of the matrix $\hat{\rho}(t)$. The von Neumann entropy predicts the upper bound of the information that we will gain after we measure a quantum state. This gain of information after measurement corresponds to the amount of information of a state. The value of this entropy stands for the degree of entanglement between the qubit and the reservoir. It has a maximal value $S_{\max }=\log (d), d$ being the dimension of the matrix $\hat{\rho}(t)$, for the maximally mixed state and is zero for the pure state.

$\frac{\text { With the eigenvalues } \lambda_{ \pm}}{\sqrt{\left.1-4 \cos ^{4}\left(\theta_{0} / 2\right)\left[\left|u_{p}(t)\right|^{2}-\left|u_{p}(t)\right|^{4}\right]\right\}} \text { of the matrix in }}$
(10), we show the von Neumann entropy in Figure 3(b) for the initially excited qubit $\left(\theta_{0}=0\right)$. The entropy has its minimal value zero at $t=0$ and reaches its maximal value $\log 2=0.693$ instantly. After a time on the order of the decay rate, the entropy becomes steady and its value is nonzero for a QPAB in the non-Markovian system $(\delta / \beta<0)$ and zero for a usual qubit in the Markovian system $(\delta / \beta=2)$. These results show that the initially purified qubit becomes maximally mixed with the reservoir instantly. As the qubit equilibrates with the $\mathrm{PhC}$ reservoir, the system becomes less mixed. A usual qubit returns to its initially purified state as it quickly disentangles from the reservoir. However, for a $\mathrm{QPAB}$, the long-time correlation with the $\mathrm{PhC}$ reservoir 
leads to the preservation of entanglement between the QPAB and its reservoir.

Combining the results of Figures 3(a) and 3(b), we infer that the coherence information of a qubit is partially lost and preserved at the very beginning and on the long run, respectively. In the QPAB of a non-Markovian case, the interaction with the $\mathrm{PhC}$ reservoir does not result in the total loss of the coherence but in the preservation of partial coherence instead. The structured reservoir of a $\mathrm{PhC}$ reveals its unique property in controlling the coherence of a qubit. A usual qubit in PhCs exhibits a short-time coherence. But a QPAB shows a property with strongly suppressed decoherence through the steady oscillation of polarization and long-time preservation of the mixed state. The greatly lowered probability of a QPAB scattered by incoherent photons of the reservoir preserves the coherence and information of a QPAB.

When we perform a multistage computation on the available information through the Markovian chain, the data processing inequality of von Neumann entropy, a basic inequality of information theory, states that the information about the output of the computation will decrease with time if the qubit storing the information is correlated with the Markovian environment [39]. This statement agrees with the result we obtained here. In the Markovian system, the newly produced mixed state by the correlation between the qubit and reservoir recovers its initially pure state and loses the information stored in the qubit. The pieces of information yielded by the multistage computations will thus be independent of each other. On the other hand, in the non-Markovian system, the newly produced mixed state will preserve the information about its output of the previous stage. The memory effect of the $\mathrm{PhC}$ reservoir on the qubit's previous state leads to the preservation of the stored information in the mixed-state qubit. Further computation operations on this mixed state can be used to increase the amount of mutual information between the outputs of the operations and the previous-stage information about the qubit state.

2.3. Reservoirs with Forbidden Bands. Now we discuss the systems suitable for realizing the behavior of strongly suppressed relaxation and decoherence. Besides the anisotropic $\mathrm{PhC}$ discussed in this section, reservoirs with forbidden bands in their dispersion relation or threshold energy in photonic DOS can be applied to exhibit these properties. Here we briefly introduce the other three systems with this kind of reservoirs including the omnidirectional waveguides (ODWGs), tunable artificial crystals, and frequency dispersive media.

An ODWG, a waveguide structure based on the complete reflection of one-dimensional (1D) PhCs, consists of a dielectric (or air) layer of lower index $n_{a}$ sandwiched by a $1 \mathrm{D} \mathrm{PhC}$ with refractive indices $n_{2}$ and $n_{1}>n_{a}$ and variable thicknesses $h_{2}, h_{1}$, and $h_{a}[17,40,41]$. This structure can exhibit complete reflection of radiation in a given frequency range for all incident angles and polarizations which have been proven both theoretically and experimentally [42-44]. Within the frequency range of omnidirectional reflection, where the dispersion curve lies inside the photonic band gap of the $\mathrm{PhC}$, the electromagnetic (EM) wave is allowed to propagate in this waveguide structure with high transmission. On the other hand, if the EM wave has its frequency lying outside this frequency range of omnidirectional reflection, it will find no propagation mode so that it cannot transmit freely through this waveguide. With an embedded qubit with frequency lying inside this forbidden range of the ODWG, the system will exhibit the properties of strongly suppressed relaxation and decoherence.

The second reservoir we introduce, a tunable artificial crystal, is constructed by periodic arrays of circuit elements of Josephson junction [45]. As the artificial crystals are built, the interaction of the EM wave with these crystals can produce a forbidden region in the band structure and DOS $[19,46]$. The frequency range of this forbidden band is tunable through the array parameters and applying external flux. When a qubit is placed in the middle of such an array of artificial crystals with its frequency lying inside the forbidden band where no traveling modes are available, this system will show the properties of strongly suppressed relaxation and decoherence.

In contrast to the PhCs, frequency dispersive media such as semiconductors and dielectrics have energy gaps caused by photon couplings to a medium excitation, for example, an exciton, optical photon, and so forth [47]. The spectrum of these media consists of two branches of allowed states separated by a gap in which propagating polariton modes are completely forbidden. Studying the quantum electrodynamics of a two-level atom placed in this medium in [20, 23], Rupasov and Singh found that if the atomic resonance frequency lies within the gap, the spectrum of the system contains a polariton-atom bound state with an eigenfrequency lying within the gap. The radiation and medium polarization of the bound state are localized in the vicinity of the atom. They predicted that the SE of this polariton-atom system is significantly suppressed due to the presence of the bound state [20]. These results about the polariton-atom bound state in the dispersive media are similar to the photon-atom bound state occurring in the $\mathrm{PhCs}$. The suppression of relaxation and decoherence rates of a QPAB is also valid for a qubit with its frequency lying inside the polariton gap in a dispersive medium.

\section{Conclusion}

A structured reservoir of an anisotropic $\mathrm{PhC}$ is introduced for suppressing the relaxation and decoherence of an embedded qubit. The dynamics of the qubit are obtained by applying fractional calculus to solve the fractional Langevin equation of the system analytically. There is a long-time memory effect being characterized by the occurrence of a negative value of the energy-relaxation rate on the dynamics of the relaxation and decoherence of a qubit. The energy-relaxation rates of QPABs and usual qubits decay to zero very quickly and slowly, respectively. The polarization and entropy of a QPAB are oscillating and decaying to a finite value, respectively. The fast decaying of the energy-relaxation rate reveals the relaxation property of a qubit being suppressed while the polarization 
oscillation and nonzero steady values of entropy manifest the decoherence being suppressed. When the dynamical behavior of the qubit formed by a photon-atom bound state is compared with that formed without forming a bound state, the suppressing effect on the relaxation and decoherence of a $\mathrm{QPAB}$ is enhanced. Physical reasons for the suppressing effect of the structured reservoir are discussed. Other systems suitable for the realization of this kind of structured reservoirs are briefly illustrated, such as the omnidirectional waveguides, tunable artificial crystals, and frequency dispersive media.

\section{Acknowledgments}

The authors acknowledge the financial support from the National Science Council (NSC) of the Republic of China under Contract nos. NSC102-2112-M-034-001-MY3 and NSC102-2112-M-009-016-MY3. S.-C. Cheng thanks the support of the National Center for Theoretical Sciences of Taiwan during visiting the center.

\section{References}

[1] L. Mazzola, S. Maniscalco, J. Piilo, K.-A. Suominen, and B. M. Garraway, "Sudden death and sudden birth of entanglement in common structured reservoirs," Physical Review A, vol. 79, no. 4, Article ID 042302, 2009.

[2] S. Maniscalco, F. Francica, R. L. Zaffino, N. Lo Gullo, and F. Plastina, "Protecting entanglement via the quantum zeno effect," Physical Review Letters, vol. 100, no. 9, Article ID 090503, 2008.

[3] B. Bellomo, R. Lo Franco, S. Maniscalco, and G. Compagno, "Entanglement trapping in structured environments," Physical Review A, vol. 78, no. 6, Article ID 060302, 2008.

[4] M. R. Jorgensen, J. W. Galusha, and M. H. Bartl, "Strongly modified spontaneous emission rates in diamond-structured photonic Crystals," Physical Review Letters, vol. 107, no. 14, Article ID 143902, 2011.

[5] S. John and T. Quang, "Spontaneous emission near the edge of a photonic band gap," Physical Review A, vol. 50, no. 2, pp. 17641769, 1994.

[6] J.-N. Wu, C.-H. Huang, S.-C. Cheng, and W.-F. Hsieh, "Spontaneous emission from a two-level atom in anisotropic oneband photonic crystals: a fractional calculus approach," Physical Review A, vol. 81, no. 2, Article ID 023827, 2010.

[7] M. M. Wolf, J. Eisert, T. S. Cubitt, and J. I. Cirac, "Assessing nonmarkovian quantum dynamics," Physical Review Letters, vol. 101, no. 15, Article ID 150402, 2008.

[8] H.-P. Breuer, E.-M. Laine, and J. Piilo, "Measure for the degree of non-Markovian behavior of quantum processes in open systems," Physical Review Letters, vol. 103, no. 21, Article ID 210401, 2009.

[9] Á. Rivas, S. F. Huelga, and M. B. Plenio, "Entanglement and nonMarkovianity of quantum evolutions," Physical Review Letters, vol. 105, no. 5, Article ID 050403, 2010.

[10] X.-M. Lu, X. Wang, and C. P. Sun, "Quantum Fisher information flow and non-Markovian processes of open systems," Physical Review A, vol. 82, no. 4, Article ID 042103, 2010.

[11] P. Haikka, S. McEndoo, G. De Chiara, G. M. Palma, and S. Maniscalco, "Quantifying, characterizing, and controlling information flow in ultracold atomic gases," Physical Review A, vol. 84, no. 3, Article ID 031602, 2011.
[12] A. M. Steane, "Error correcting codes in quantum theory," Physical Review Letters, vol. 77, no. 5, pp. 793-797, 1996.

[13] D. Bacon, D. A. Lidar, and K. B. Whaley, "Robustness of decoherence-free subspaces for quantum computation," Physical Review A, vol. 60, no. 3, pp. 1944-1955, 1999.

[14] L. Viola and S. Lloyd, "Dynamical suppression of decoherence in two-state quantum systems," Physical Review A, vol. 58, no. 4, pp. 2733-2744, 1998.

[15] L.-A. Wu and D. A. Lidar, "Creating decoherence-free subspaces using strong and fast pulses," Physical Review Letters, vol. 88, no. 20, pp. 2079021-2079024, 2002.

[16] H. K. Ng, D. A. Lidar, and J. Preskill, "Combining dynamical decoupling with fault-tolerant quantum computation," Physical Review A, vol. 84, no. 1, Article ID 012305, 2011.

[17] C.-H. Huang, S.-C. Cheng, J.-N. Wu, and W.-F. Hsieh, "Spontaneous emission dynamics in an omnidirectional waveguide made of photonic crystals," Journal of Physics Condensed Matter, vol. 23, no. 22, Article ID 225301, 2011.

[18] S. Savel'ev, A. L. Rakhmanov, and F. Nori, "Using Josephson vortex lattices to control terahertz radiation: tunable transparency and terahertz photonic crystals," Physical Review Letters, vol. 94, no. 15, Article ID 157004, 2005.

[19] C. Hutter, E. A. Tholén, K. Stannigel, J. Lidmar, and D. B. Haviland, "Josephson junction transmission lines as tunable artificial crystals," Physical Review B, vol. 83, no. 1, Article ID 014511, 2011.

[20] V. I. Rupasov and M. Singh, "Polariton-atom bound state in a dispersive medium," Physics Letters A, vol. 222, no. 4, pp. 258262, 1996.

[21] A. Hatef and M. R. Singh, "Plasmonic effect on quantum coherence and interference in metallic photonic crystals doped with quantum dots," Physical Review A, vol. 81, no. 6, Article ID 063816, 2010.

[22] M. R. Singh, C. Racknor, and D. Schindel, "Controlling the photoluminescence of acceptorand donor quantum dots embedded in a nonlinear photonic crystal," Applied Physics Letters, vol. 101, Article ID 051115, 2012.

[23] V. I. Rupasov and M. Singh, "Quantum gap solitons and many-polariton-atom bound states in dispersive medium and photonic band gap," Physical Review Letters, vol. 77, no. 2, pp. 338-341, 1996.

[24] M. R. Singh and W. Lau, "Polariton spontaneous emission superradiance and polariton-impurity states in III-V semiconductors," Physics Letters A, vol. 231, no. 1-2, pp. 115-122, 1997.

[25] M. R. Singh, "Controlling spontaneous emission in photonicband-gap materials doped with nanoparticles," Physical Review A, vol. 75, no. 3, Article ID 033810, 2007.

[26] M. R. Singh, "Photon transparency in metallic photonic crystals doped with an ensemble of nanoparticles," Physical Review A, vol. 79, no. 1, Article ID 013826, 2009.

[27] S.-Y. Zhu, H. Chen, and H. Huang, "Quantum interference effects in spontaneous emission from an atom embedded in a photonic band gap structure," Physical Review Letters, vol. 79, no. 2, pp. 205-208, 1997.

[28] S.-C. Cheng, J.-N. Wu, M.-R. Tsai, and W.-F. Hsieh, "Spontaneous emission near the band edge of a three-dimensional photonic crystal: a fractional calculus approach," Journal of Physics Condensed Matter, vol. 21, no. 1, Article ID 015503, 2009.

[29] S. John and T. Quang, "Collective switching and inversion without fluctuation of two-level atoms in confined photonic systems," Physical Review Letters, vol. 78, no. 10, pp. 1888-1891, 1997. 
[30] A. G. Kofman, G. Kurizki, and B. Sherman, "Spontaneous and induced atomic decay in photonic band structures," Journal of Modern Optics, vol. 41, p. 353, 1994.

[31] S. Ogawa, M. Imada, S. Yoshimoto, M. Okano, and S. Noda, "Control of light emission by 3D photonic crystals," Science, vol. 305, no. 5681, pp. 227-229, 2004.

[32] P. Lodahl, A. F. Van Driel, I. S. Nikolaev et al., "Controlling the dynamics of spontaneous emission from quantum dots by photonic crystals," Nature, vol. 430, no. 7000, pp. 654-657, 2004.

[33] N. Vats, S. John, and K. Busch, "Theory of fluorescence in photonic crystals," Physical Review A, vol. 65, no. 4 B, pp. 438081-4380813, 2002.

[34] S. John, "Strong localization of photons in certain disordered dielectric superlattices," Physical Review Letters, vol. 58, no. 23, pp. 2486-2489, 1987.

[35] R. R. Nigmatullin, "Fractional integral and its physical interpretation," Theoretical and Mathematical Physics, vol. 90, no. 3, pp. 242-251, 1992.

[36] I. Podlubny, Fractional Differential Equations, Academic Press, New York, NY, USA, 1999.

[37] A. A. Stanislavsky, "Fractional oscillator," Physical Review E, vol. 70, no. 5, Article ID 051103, 6 pages, 2004.

[38] K. S. Miller and B. Ross, An Introduction to the Fractional Calculus and Fractional Differential Equations, John Wiley \& Sons, New York, NY, USA, 1993.

[39] M. A. Nielsen and I. L. Chuang, Quantum Computation and Quantum Information, Cambridge University Press, Cambridge, UK, 2000.

[40] B. Chen, T. Tang, Z. Wang, H. Chen, and Z. Liu, "Flexible optical waveguides based on the omnidirectional reflection of one-dimensional photonic crystals," Applied Physics Letters, vol. 93, no. 18, Article ID 181107, 2008.

[41] S. G. Johnson and J. D. Joannopoulos, "Block-iterative frequency-domain methods for Maxwell's equations in a planewave basis," Optics Express, vol. 8, no. 3, pp. 173-190, 2001.

[42] Y. Fink, J. N. Winn, S. Fan et al., "A dielectric omnidirectional reflector," Science, vol. 282, no. 5394, pp. 1679-1682, 1998.

[43] J. N. Winn, Y. Fink, S. Fan, and J. D. Joannopoulos, "Omnidirectional reflection from a one-dimensional photonic crystal," Optics Letters, vol. 23, no. 20, pp. 1573-1575, 1998.

[44] S.-H. Kim and C. K. Hwangbo, "Design of omnidirectional high reflectors with quarter-wave dielectric stacks for optical telecommunication bands," Applied Optics, vol. 41, no. 16, pp. 3187-3192, 2002.

[45] J. Q. You and F. Nori, "Atomic physics and quantum optics using superconducting circuits," Nature, vol. 474, no. 7353, pp. 589597, 2011.

[46] A. L. Rakhmanov, A. M. Zagoskin, S. Savel'ev, and F. Nori, "Quantum metamaterials: electromagnetic waves in a Josephson qubit line," Physical Review B, vol. 77, no. 14, Article ID 144507, 2008.

[47] V. M. Agranovich and V. L. Ginzburg, Crystal Optics with Spatial Dispersion, and Excitons, Springer, Berlin, Germany, 1984. 

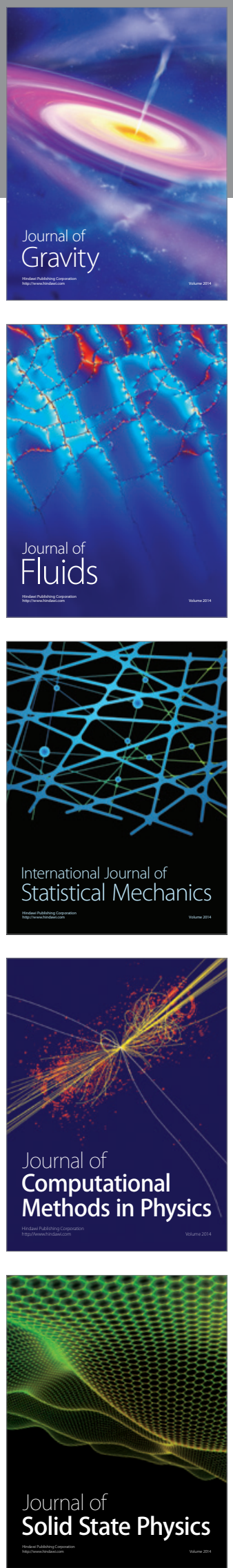

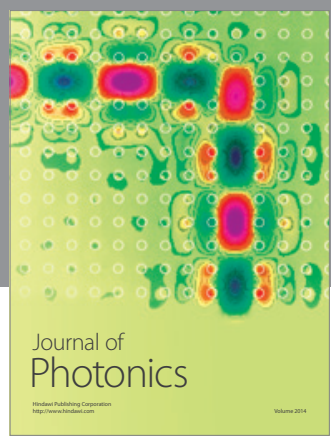

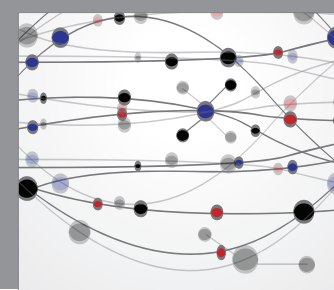

The Scientific World Journal

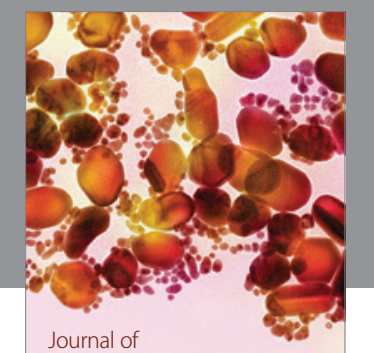

Soft Matter
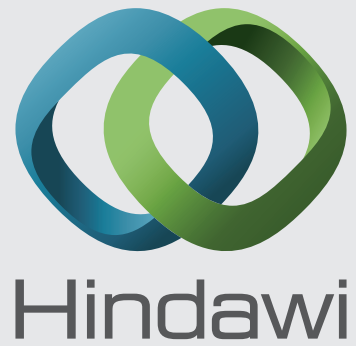

Submit your manuscripts at

http://www.hindawi.com
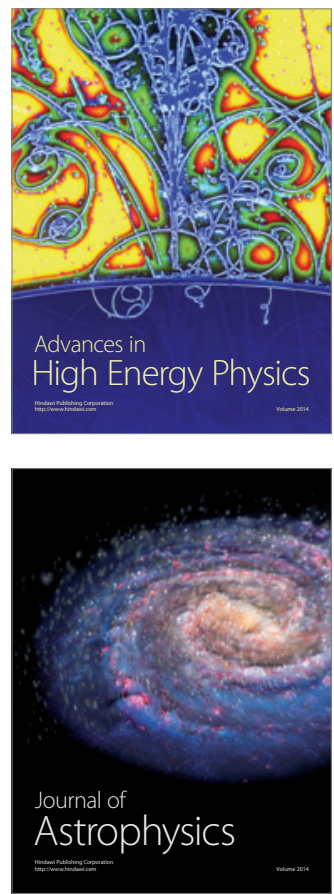
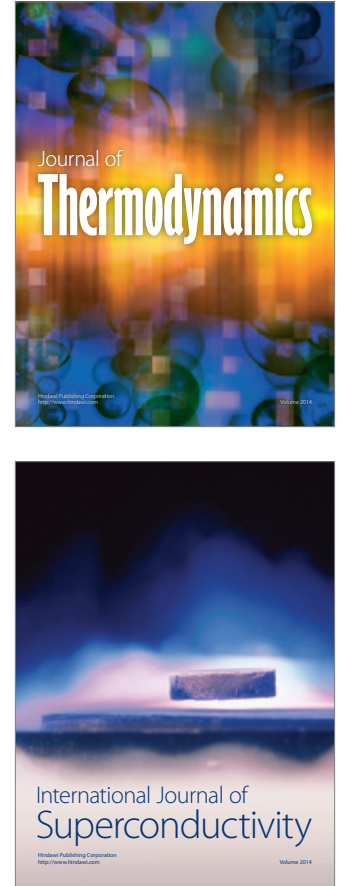
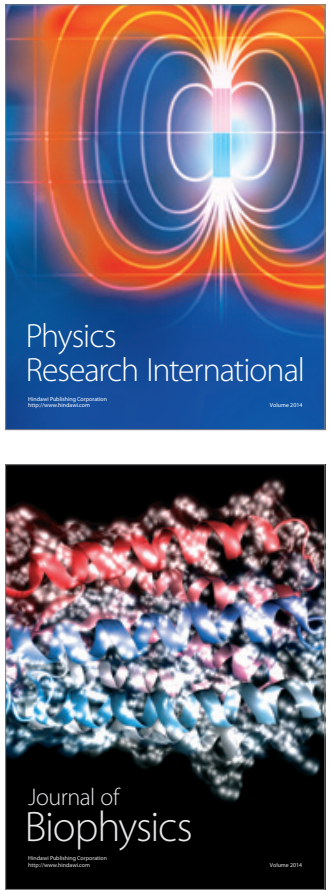
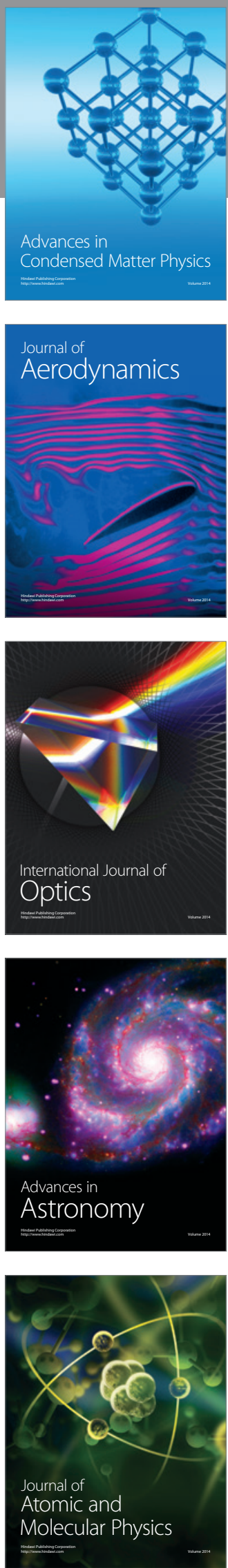